\title{
Development of a high-speed, reciprocating electrostatic probe system for Hall thruster interrogation
}

\author{
James M. Haas and Alec D. Gallimore ${ }^{\text {a) }}$ \\ Department of Aerospace Engineering, Plasmadynamics and Electric Propulsion Laboratory, \\ The University of Michigan, College of Engineering, Ann Arbor, Michigan 48109 \\ Keith McFall and Greg Spanjers \\ Air Force Research Laboratory, Edwards Air Force Base, California 93524
}

(Received 18 April 2000; accepted for publication 15 August 2000)

\begin{abstract}
The use of electrostatic probes to measure local plasma parameters inside the discharge chamber of a Hall thruster presents significant difficulties. The high-temperature, dense plasma, and Hall current in the accelerating channel heat the probe rapidly causing ablation of probe material, which perturbs thruster operation and reduces probe lifetime. Results are presented which show the extent of perturbation to discharge current, cathode potential, and thrust for the case where probe material is ablated. A simple thermal model of probe material heating is developed and ablation times for a typical probe configuration are presented. Using the results of the thermal model, a high-speed axial reciprocating probe (HARP) system was developed to enable probe survival and reduce thruster perturbations during interrogation of the discharge chamber of a Hall thruster. Results using the HARP system are presented showing a significant reduction in thruster perturbation. The results also indicate that a mechanism other than material ablation is contributing to perturbation of the thruster. Based on emissive probe data, the tungsten conductor appears to provide a low impedance path between magnetic field lines, enhancing electron transport to the anode. (C) 2000 American Institute of Physics. [S0034-6748(00)04211-8]
\end{abstract}

\section{INTRODUCTION}

The need for accurate plasma parameter measurements inside the Hall thruster, also referred to as a closed drift thruster (CDT), has grown appreciably in recent years as the focus of research has shifted from plume/spacecraft interactions to understanding the basic physical processes of thruster operation. The CDT is a coaxial device in which orthogonal electric and magnetic fields are employed to ionize an inert gas, such as xenon, through electron impact and accelerate the resulting ions to produce thrust. The main components of the CDT are shown in Fig. 1. The magnetic field that is produced by an electromagnet is channeled between an inner ferromagnetic core (pole piece) and outer ferromagnetic ring. The electromagnet circuit is usually composed of three or four outer magnet coils and one central coil, although permanent magnets have been used for smaller devices. Though separate power supplies can be used to energize the electromagnet coils, the number of turns in each is usually set so the electromagnet establishes the proper magnetic field strength when it is operated in series with the discharge supply. This configuration results in an approximately radial magnetic field with a peak strength of a few hundred Gauss near the exit of the engine. The field strength is such that only the electrons are magnetized. The radial magnetic field exhibits a bell-shaped axial profile with the peak situated near the exit. Most of the acceleration takes place near the region of peak magnetic field strength.

\footnotetext{
a) Author to whom correspondence should be addressed; electronic mail: haas@engin.umich.edu
}

An axial electric field is provided by applying a voltage between the anode and the cathode. As the electrons stream upstream toward the anode from the cathode, their motion is impeded by the magnetic field. Because of the orientation of the electric and magnetic fields, the electrons drift in the $\mathbf{E} \times \mathbf{B}$ direction, forming an azimuthal Hall current. These electrons also ionize propellant molecules that are injected through the anode and which are subsequently accelerated by the axial electric field. From an electrostatic point of view, the magnetic field suppresses the axial mobility of the electrons while exerting essentially no effect on the ions. This enables the plasma to support an axial electric field with a potential difference close to the applied voltage between the electrodes. Thus, the bulk of the ions are accelerated to kinetic energies within $80 \%$ of the applied discharge voltage. Moreover, the mixture of electrons and ions in the acceleration zone means that the plasma is electrically neutral, and as such, is not space-charge limited in ion current density as in the case of a gridded ion thruster. This combination of processes accounts for the CDT's high thrust efficiency and thrust density. The electromagnetic point of view is simply that the azimuthal Hall current interacts with the radial applied magnetic field and accelerates the plasma axially through the $\mathbf{J} \times \mathbf{B}$ Lorentz force.

While the basic operation of the CDT has been well characterized and the thrusters employed successfully onboard spacecraft, a better understanding of the ionization and acceleration mechanisms is needed in order to improve the performance of next generation Hall thrusters. This information will also play an invaluable role in the validation of 


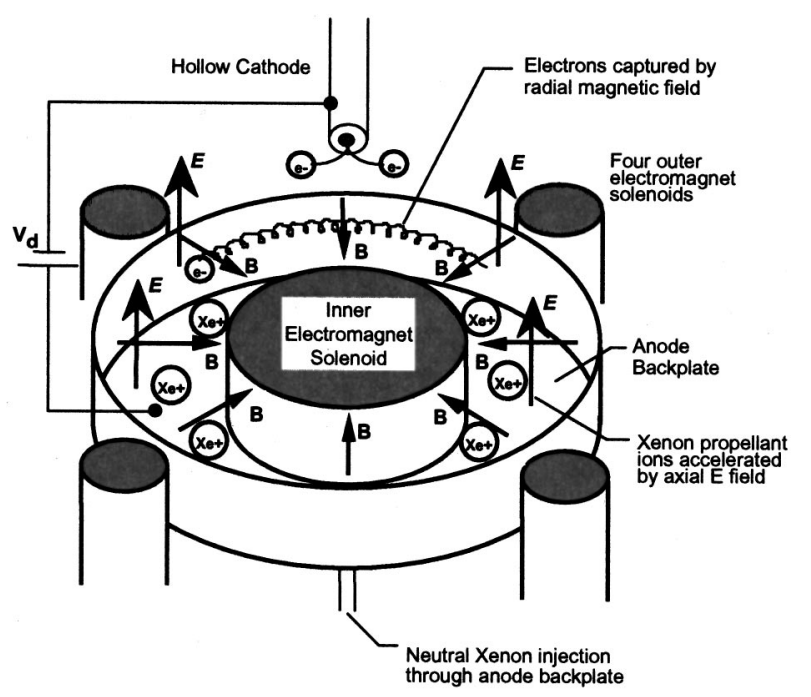

FIG. 1. Schematic showing typical CDT construction.

existing and future numerical models. The increasing time and expense associated with developing new thrusters makes it imperative that accurate and reliable predictive models are developed.

Electrostatic (or Langmuir) probes are among the simplest and most commonly used plasma diagnostic tools. Their use dates back to Langmuir's ${ }^{1}$ original development of the theory to describe probe behavior in a plasma. Since that time electrostatic probes have found use in a wide range of disciplines ranging from nuclear fusion ${ }^{2}$ to atmospheric and space sciences. ${ }^{3}$ In particular, they have been used extensively in the field of electric propulsion to provide a relatively simple means of measuring electron temperature, electron number density, plasma potential, and floating potential. Electrostatic probes have been used in single, double, triple, and quadruple configurations in a number of devices including arcjets, Lorentz force accelerators, ion engines, and Hall thrusters. ${ }^{4-11}$

In plasmas with highly energetic charged particles, probe material is generally sputtered and/or ablated by direct particle flux on very small time scales. The local plasma temperature and density may then be modified through emission of relatively cold probe material. These perturbations may remain localized near the probe, or may propagate further into the plasma affecting the macroscopic properties of the plasma being interrogated. As time scales increase, probe survival becomes an issue. In the case of Hall thrusters, for measurements downstream of the exit plane, probe survival is not a concern nor does the presence of the probe adversely affect thruster operation. However, within the discharge chamber plasma temperature and density increase, which leads to increased probe heating. Moreover, the presence of an intense Hall current in the acceleration region of the Hall thruster further exacerbates this problem. Probe material ablation then becomes significant and survival times are shortened drastically. Under these conditions, a high speed, reciprocating probe system can reduce the amount of ablated material, allowing for more accurate measurements of local plasma parameters and assuring probe survival.

This work presents efforts to quantify the extent of per- turbations on thruster operation resulting from probe material ablation. A thermal model of probe heating resulting from high-energy particle impact in the Hall thruster discharge chamber is presented and its results and implications for maximum residence times are discussed. A high-speed axial reciprocating probe (HARP) system, based on data from the thermal model, was built and the results of its use are presented.

\section{EXPERIMENTS TO QUANTIFY PROBE ABLATION EFFECTS}

The initial step in developing a probe diagnostic to reduce perturbative effects was to determine what influences ablating probe material had on thruster operation. This was accomplished through a series of experiments conducted at the Air Force Research Laboratory (AFRL).

\section{A. AFRL test facility}

All experiments were conducted in Chamber 6 at AFRL. This $2 \mathrm{~m}$ diam by $3 \mathrm{~m}$ long cryopumped vacuum facility had a total pumping speed of $26000 \mathrm{l} / \mathrm{s}$ of xenon and a base pressure of $2.7 \times 10^{-6}$ Torr. Background chamber pressure was maintained below $5 \times 10^{-5}$ Torr, corrected for xenon, during all tests. This facility has been discussed in detail in a previous work. ${ }^{12}$ The setup for this experiment consisted of an SPT-70 mounted to a Hall thruster thrust stand and a “dummy" probe mounted to a linear $X-Y$ positioning system to investigate the effects of probe material ablation on thruster operation. The probe was constructed of tungsten wire and an alumina insulator. The probe tip was imaged onto the entrance slit of a SPEX $1 \mathrm{~m}$ spectrometer that was used to monitor emission from the ablating probe material. A Tektronix TDS 420 A digital oscilloscope simultaneously recorded output from the spectrometer, thruster discharge current, cathode potential, and output from the thrust stand. In this way, perturbations to the various thruster parameters could be monitored as a function of probe ablation.

\section{B. Thruster}

A Fakel SPT-70 was used for all experiments at AFRL. This thruster was designed to operate at $660 \mathrm{~W}$ yielding 40 $\mathrm{mN}$ of thrust and $1500 \mathrm{~s}$ specific impulse at $40 \%$ efficiency. ${ }^{12}$ For all tests the SPT-70 was operated at $300 \mathrm{~V}$, $2.2 \mathrm{~A}$, and $26 \mathrm{sccm}$ of xenon, $3 \mathrm{sccm}$ of which was supplied to the cathode.

\section{Results}

Figure 2 illustrates the effect of probe material ablation on the operational characteristics of the thruster. No attempt was made to avoid material ablation since the purpose of this experiment was to determine the effect probe ablation has on thruster operation. The probe was simply inserted into the thruster for an extended period of time to study both the transient and steady state effects. The bottom plot is the output from the spectrometer and shows clearly when the probe began to ablate. Discharge current initially increased by $50 \%$ then dropped to a value approximately $20 \%$ above the nominal level. Cathode potential behaved similarly, increasing 


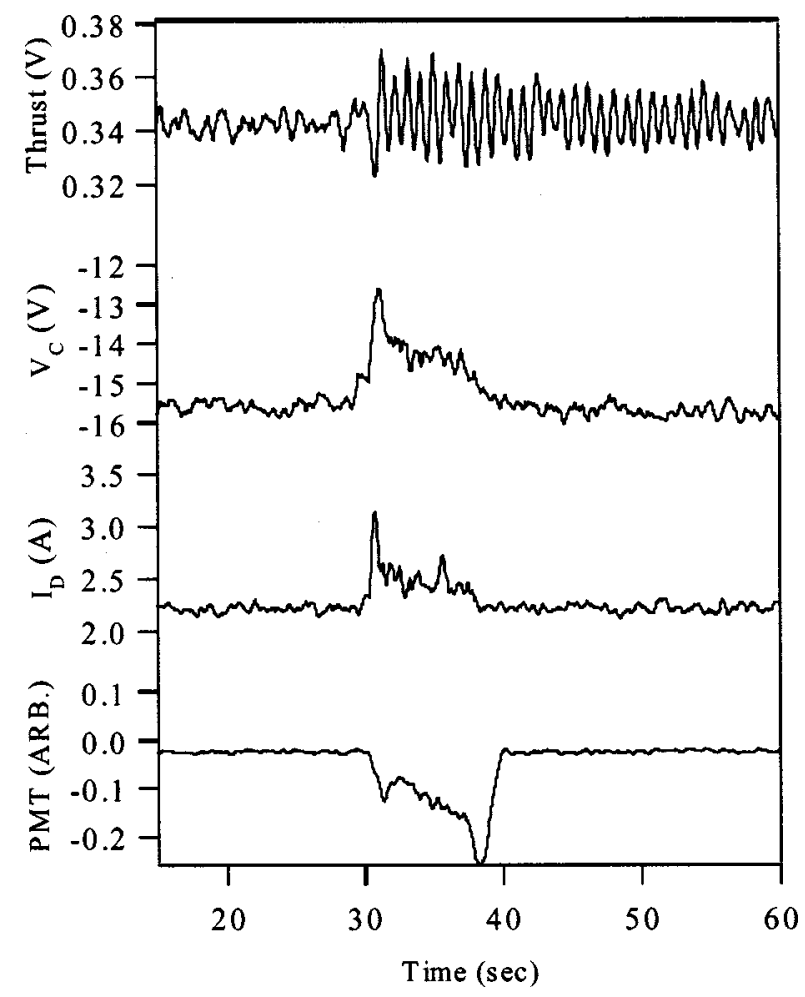

FIG. 2. SPT-70 thruster parameter perturbation from a previous study.

initially by $20 \%$ then leveling off approximately $10 \%$ above nominal. The top plot in Fig. 2 shows the oscillation induced in the thrust. The continuing oscillation after the probe ceased ablating material is a result of the thrust stand being underdamped.

These experiments also yielded an unexpected result. Upon examination of the "dummy" probe after the test was completed, it appeared that the probe had experienced very localized ablation several millimeters upstream of the tip, as illustrated in Fig. 3. This implied that the heating mechanism varied along the axis of the discharge chamber, with greater heating occurring at a location several millimeters upstream of the exit plane. This is the general location of the peak electric field in Hall thrusters of this type ${ }^{13}$ (i.e., thrusters with a ceramic discharge chamber) and it follows that this would be the location of maximum Hall current. This apparent dominance of the Hall current as a probe heating mechanism is supported by the results of the thermal model presented in Sec. III.

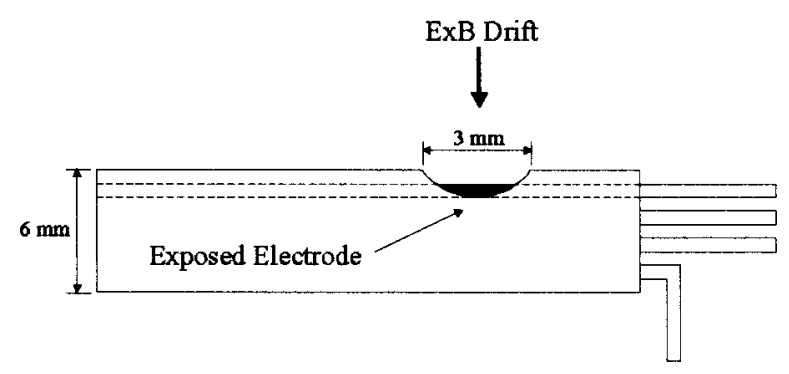

FIG. 3. Illustration of probe ablation from ARFL study.

\section{ELECTROSTATIC PROBE THERMAL MODEL}

Once the effects of ablating probe material were better understood, a simple model was developed to predict the maximum residence time of an electrostatic probe inside the Hall thruster before the onset of ablation. While the initial experiments of the previous section were conducted using an SPT-70 operating at $660 \mathrm{~W}$, the ultimate goal was to make measurements inside a $5 \mathrm{~kW}$ laboratory-model Hall thruster. Thus the parameters in the model presented below reflect conditions expected in a $5 \mathrm{~kW}$ thruster. Initially, the probe was modeled as a cylinder with radius $r$ and length $l$ with heat conduction along the length of the probe taken into account. However, because of the short time scales involved, it was determined that heat conduction was negligible and so a simplified, "bulk" heating approach was used. The model assumed that all probe heating resulted from particle flux to the surface and consisted of both a directed component dictated by the thruster parameters and a random component dictated by the dynamics of the sheath surrounding the probe. The total particle flux was further subdivided into the contribution from the electrons and ions. Equation (1) is the power density to the probe, which is expressed as the sum of the products of the flux of each particle species $j$ and its kinetic energy

$$
\left.\frac{P}{A}\right|_{\text {total }}=\sum_{j} K_{j} f_{j}=\frac{1}{2} m_{j} v_{j}^{2} f_{j} .
$$

The four components of the total power density seen by the probe consist of the following:

(1) Directed ion flux to the probe tip where

$$
\begin{aligned}
& f=n_{1} \nu, \\
& \nu=\sqrt{\frac{2 E e}{m_{i}}} .
\end{aligned}
$$

(2) Random ion flux through the sheath where

$$
\begin{aligned}
& f=n_{e 0} \nu, \\
& \nu=\sqrt{\frac{k_{b} T_{e}}{m_{i}}} .
\end{aligned}
$$

(3) Random electron flux through the sheath where

$$
\begin{aligned}
& f=\frac{1}{4} n_{e} \nu, \\
& n_{e}=n_{e 0} \exp \left[\frac{e\left(V_{f}-V_{p}+\Delta V\right)}{k_{b} T_{e}}\right], \\
& \nu=\sqrt{\frac{8 k_{b} T_{e}}{\pi m_{e}}} .
\end{aligned}
$$

(4) Directed electron flux from the Hall current where

$$
\begin{aligned}
& \nu_{\mathrm{ExB}}=\frac{E}{B}, \\
& f=n_{e 0} \nu .
\end{aligned}
$$

The bulk melting time is given by Eq. (11) 


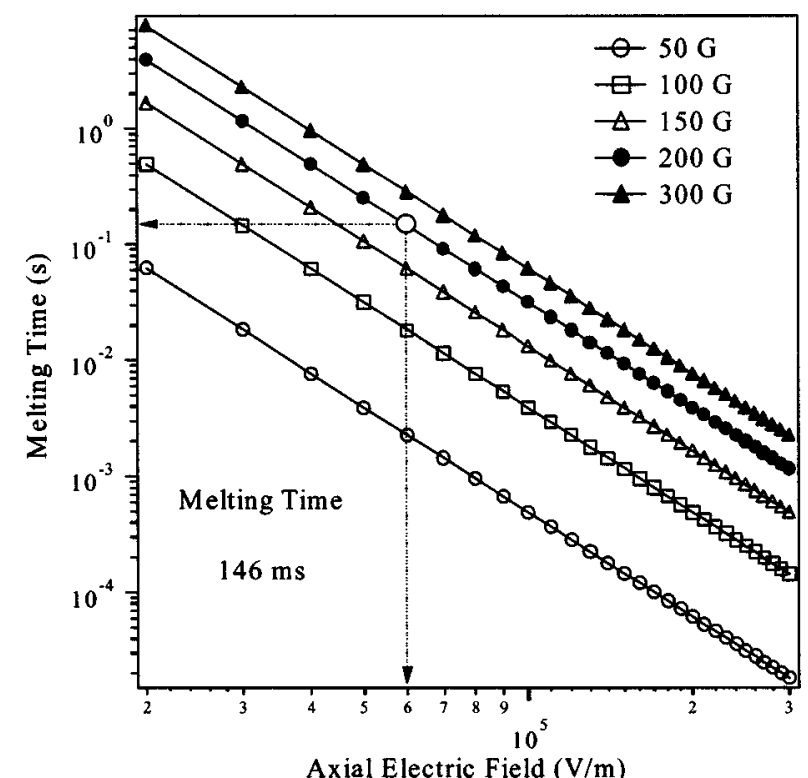

FIG. 4. Probe melting time vs axial electric field strength for several magnetic field values.

$$
t_{\mathrm{bulk}}=\frac{r \rho C_{p} T_{\max }}{2[P / A]_{\mathrm{total}}} .
$$

It was assumed that the plasma was quasineutral $\left(n_{e}\right.$ $=n_{i}=n$ ) and a conservative estimate of $5 \times 10^{18} \mathrm{~m}^{-3}$ for number density was used. The results of the thermal model indicated that the contribution of the Hall current to the total power density was at least an order of magnitude larger than each of the other components. As stated previously, this supports experimental data, which indicated the dominant heating mechanism was the Hall current. Figure 4 shows the dependence of ablation time on the axial electric field for several values of the radial magnetic field. Using representative values for a $5 \mathrm{~kW}$ Hall thruster, $E=6 \times 10^{4} \mathrm{~V} / \mathrm{m}$ and $B$ $=200 \mathrm{G}$, a predicted material ablation time of approximately $150 \mathrm{~ms}$ was calculated as shown in Fig. 4. This value became the figure of merit for the development of the high-speed electrostatic probe system discussed in Sec. IV. It should be noted that far downstream of the exit plane, where the probe resided between "sweeps" inside the thruster, plasma density and temperature were sufficiently small that probe heating was negligible.

\section{DESCRIPTION OF ELECTROSTATIC PROBE DIAGNOSTIC}

Using the results of the thermal model presented in Sec. III, the HARP system was developed. The goal of this system was to insert electrostatic probes inside the Hall thruster discharge chamber and remove them on sufficiently small time scales such that the probes would not be destroyed and that perturbation to the thruster would be reduced. The system had three main components: the high-speed actuator, the electrostatic probe, and the data acquisition subsystem. Each of these subsystems is discussed in more detail below.

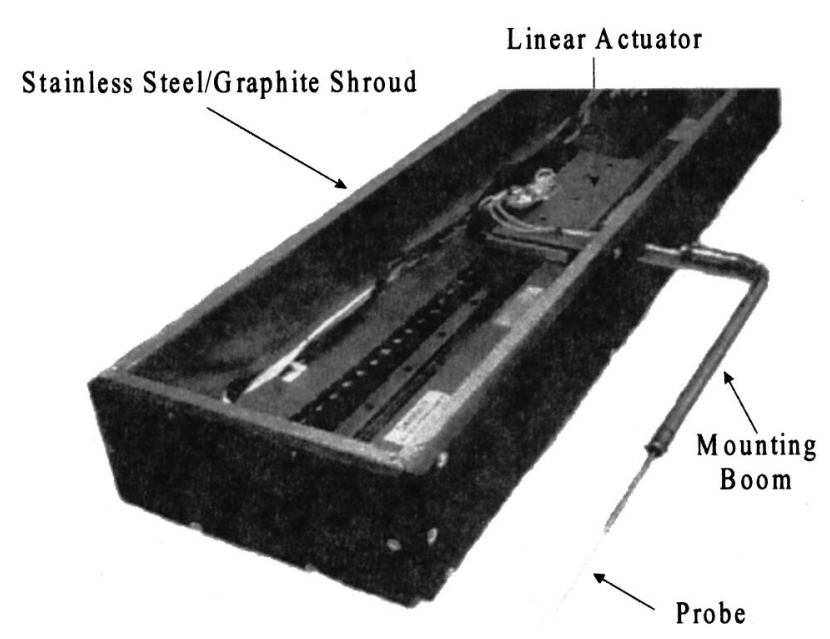

FIG. 5. Probe, actuator, and protective shroud (top cover is removed to show actuator).

\section{A. High speed actuator}

The need for high-speed probe positioners has existed in other fields for many years. External, pneumatic systems are commonly used, for example, to study edge effects in Tokamaks. ${ }^{14}$ This type of actuator proved to be infeasible for studying Hall thrusters due to the necessity of positioning the thruster as far from the walls of the vacuum chamber as possible in order to minimize contamination from sputtered material and background gases. This limitation on thruster position required that the actuator be placed inside the chamber, thus ruling out pneumatic systems. A second alternative was a conventional belt/pulley or leadscrew type assembly. These were also ruled out because they were incapable of providing the high acceleration and velocity necessary. It was determined that the best choice was a linear motor assembly providing direct linear motion at very high speed and acceleration. The linear motor selected was an LM210 manufactured by Trilogy. It was a three-phase brushless dc servo motor consisting of a linear " $U$ '"-shaped magnet track and a ' $\mathrm{T}$ ' '-shaped coil moving on a set of linear tracks. The only contact between moving parts was through the guide rails, providing very little resistance. The motor employed Sine commutation, using a linear encoder built into the magnetic track to provide position feedback for very smooth motion. The linear encoder provided positioning resolution to $5 \mu \mathrm{m}$. A Pacific Scientific SC950 digital, brushless servo drive was used to control the motor, which in turn was controlled through a PC via a serial cable. The table was covered by a stainless steel and graphite shroud to protect it from direct impact of high-energy ions and to prevent the motor from being excessively heated, which would lead to performance degradation. One side had a thin slit running the length of the table through which a probe boom extended. Figure 5 shows the HARP assembly, linear motor, protective shroud, and the boom to which an electrostatic probe is attached.

\section{B. Electrostatic probe}

Several factors were taken into consideration in the design of the electrostatic probe. While the thermal model indicated that the ablation time was effectively independent of 


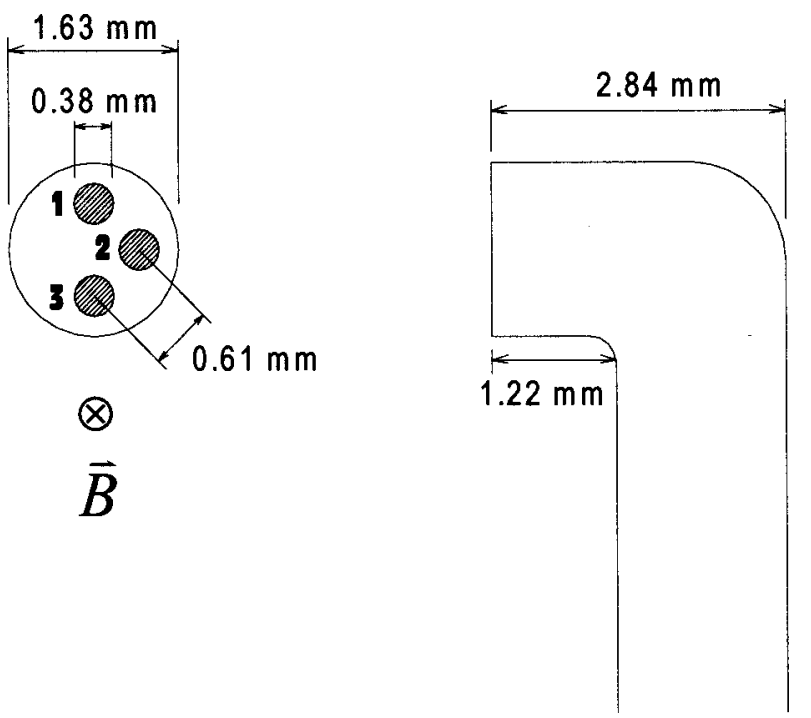

FIG. 6. Schematic of planar triple Langmuir probe.

the probe size, it was highly dependent on the material properties, particularly density and specific heat. The results presented in Fig. 4 are for standard probe material's, alumina and tungsten. No other refractory metal surpassed the thermal characteristics of tungsten. Several materials, on the other hand, were considered as a replacement for alumina as an insulator. Beryllia $(\mathrm{BeO})$ had a density and melting temperature comparable to alumina while possessing a higher value of specific heat which, referring back to Eq. (11), would result in longer ablation times. However, beryllia has a high pulmonary toxicity that makes it unsuitable for general laboratory use. Diamond was also considered because of its high specific heat but was eliminated based on its large secondary electron emission coefficient that would create significant problems in a diagnostic that depends on the collection of charged particles to measure plasma parameters. Silicon nitride possessed a high working temperature and large specific heat. However, its thermal properties offered no advantages over alumina and was not nearly as inexpensive and easily acquired in tube form. As such, it was determined that alumina was the best choice for the probe insulator.

Size was also an important consideration in probe design. The dimensions of the thruster dictated that the size should be minimized as much as possible in order to improve the spatial resolution of the measurements. The smaller size would also minimize the current collected by the probe, thus reducing perturbation to the plasma. Conversely, the characteristics of the high-speed actuator necessitated a probe large enough that it could withstand the mechanical stress of a large acceleration. Through trial and error, it was determined that a probe constructed of an alumina insulator $1.5 \mathrm{~mm}$ in diameter would meet the size and strength requirements. Two types of probes were constructed: the planar triple Langmuir probe in Fig. 6 and the emissive probe in Fig. 7. The triple probe consisted of three $0.38 \mathrm{~mm}$ diam planar electrodes insulated from each other and from the plasma inside a $1.63 \mathrm{~mm}$ diam alumina insulator. The probe was constructed with a $90^{\circ}$ bend such that the normal to the sur- $\otimes$

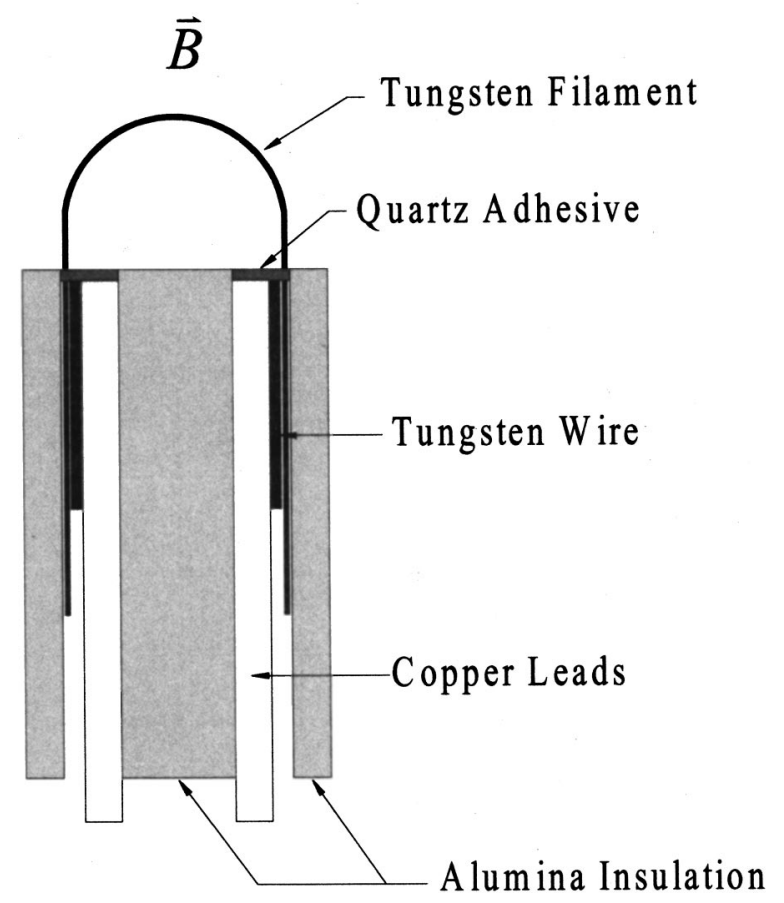

FIG. 7. Schematic of emissive probe.

face of the electrodes was oriented parallel to the magnetic field. This configuration resulted in a current collected by the probe that was composed mainly of electrons whose motion toward the probe was not impeded by the presence of the magnetic field. This significantly simplified subsequent probe data analysis. The emissive probe consisted of an emitting filament made from $0.127 \mathrm{~mm}$ diam tungsten wire. The ends of this filament were inserted approximately 76 $\mathrm{mm}$ down two bores of a $1.63 \mathrm{~mm}$ diam alumina tube along with 30 American Wire Gauge copper wire leads. Once the tungsten filament and copper leads were in place additional, shorter lengths of tungsten wire were inserted into the alumina tubing to provide a tight fit and guarantee good contact between the tungsten and copper wires. Cotronics 905 quartz adhesive, with a working temperature of $1375^{\circ} \mathrm{C}$, was used to cement the filament in place.

\section{Data acquisition}

Large potentials inside the discharge chamber created significant difficulties in making measurements. At a nominal thruster operating condition of $300 \mathrm{~V}$ the entire probe circuit floated to potentials of several hundred volts above ground. This created a potential hazard for the data acquisition hardware, which was limited to a differential voltage input of $10 \mathrm{~V}$. A voltage divider was used to reduce probe signals to an acceptable range, however, in order to measure voltages of this magnitude without significant leakage current to ground (which would cause the circuit to float at a value other than the true floating potential), resistors of several hundred megaohms were needed. This in turn required isolation between the megaohm sized resistors and the relatively low input impedance $(\sim 200 \mathrm{k} \Omega)$ of the data acquisition board. A circuit was constructed based around the Ana- 


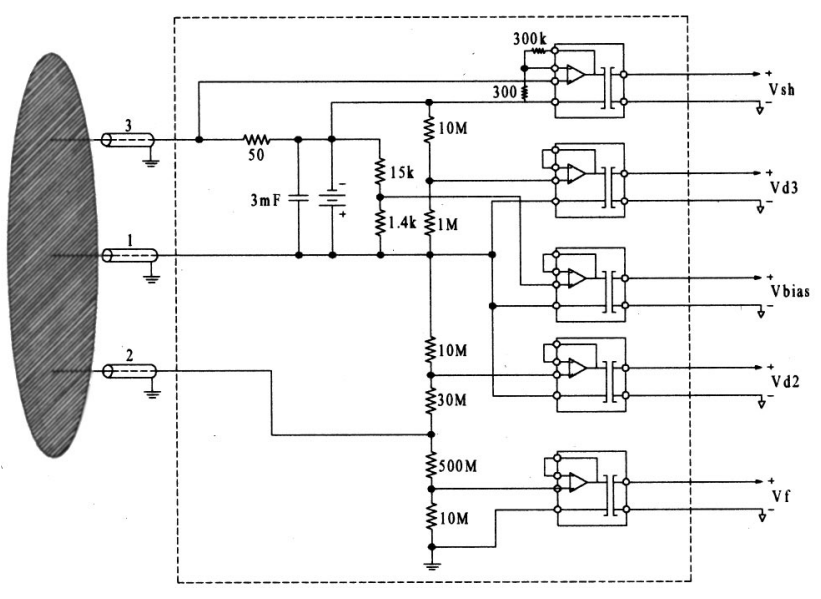

FIG. 8. Triple probe circuit.

$\log$ Devices AD210 isolation amplifier. This amplifier was capable of handling several thousand volts of common mode voltage and provided an input impedance of $1 \times 10^{14} \Omega$. The low impedance output $(\sim 1 \Omega)$ of the isolation amplifier connected to the computer-driven data acquisition board for data recording and storage. The AD210 also offered complete isolation of the probe from the data acquisition hardware through transformer coupling. Figure 8 illustrates the triple probe circuit. The floating emissive probe circuit, also constructed using the AD210 isolation amplifier, was considerably simpler and is shown in Fig. 9. All probe connections were made with coaxial feedthroughs at the chamber wall.

\section{EXPERIMENTS TO REDUCE PERTURBATIONS DURING HALL THRUSTER INTERROGATION}

Once the high-speed system, probes, and data acquisition were completed, the next step was to attempt to reduce

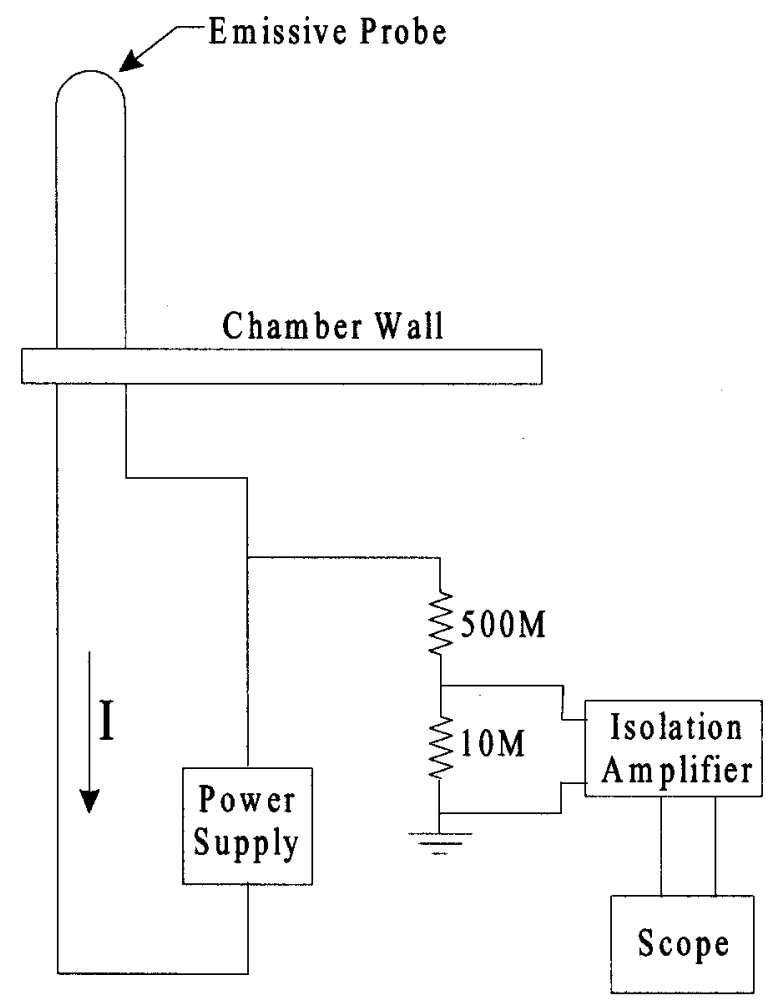

FIG. 9. Floating emissive probe circuit.

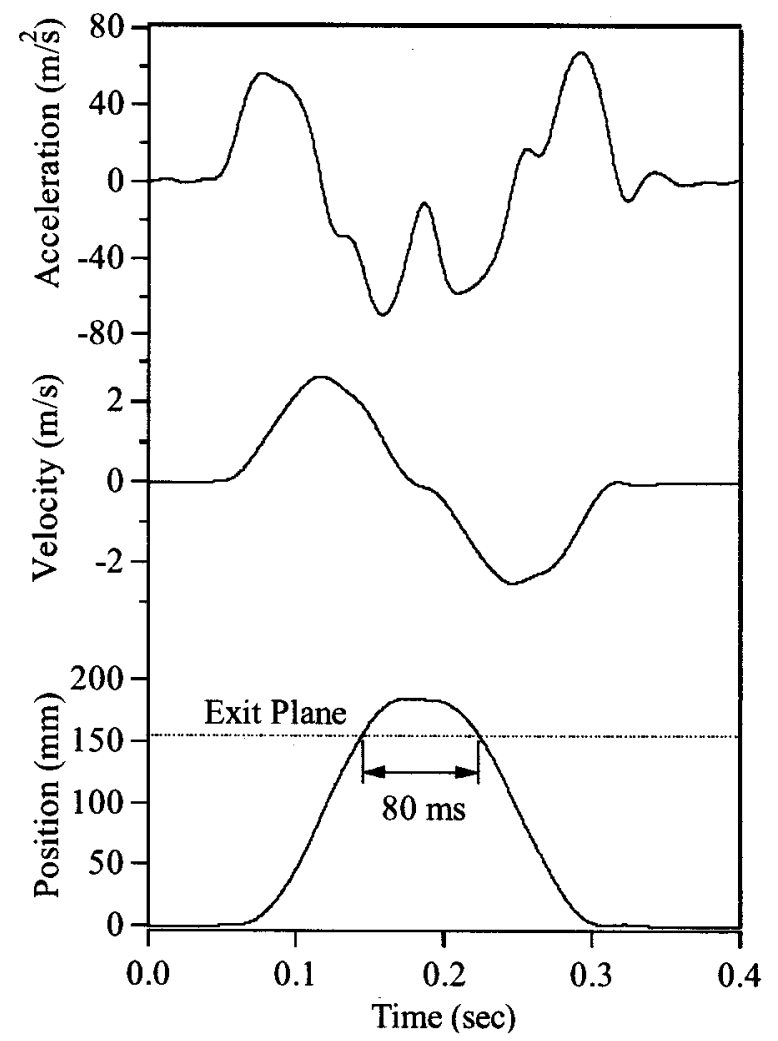

FIG. 10. Representative HARP system performance.

the perturbation to the Hall thruster while interrogating the discharge channel. This section presents the results of those experiments and discusses the implications for future internal Hall thruster measurements.

\section{A. Plasmadynamics and Electric Propulsion Laboratory (PEPL) vacuum facility}

This set of experiments was conducted in the University of Michigan PEPL $6 \mathrm{~m}$ diam by $9 \mathrm{~m}$ long large vacuum test facility (LVTF). The pumping system consisted of four CVI model TM-1200 reentrant cryopumps providing a measured xenon pumping speed of $140000 \mathrm{l} / \mathrm{s}$ and an ultimate base pressure of $2 \times 10^{-7}$ Torr. The operating pressure was 5.5 $\times 10^{-6}$ Torr at $5.4 \mathrm{~A}$ and $1.0 \times 10^{-5}$ Torr at $10 \mathrm{~A}$, when corrected for xenon. This facility has been described in detail in a previous work. ${ }^{15}$

\section{B. Thruster}

For all tests in the LVTF, the P5 $5 \mathrm{~kW}$ laboratory model Hall thruster was used. This thruster has been thoroughly characterized and its performance closely matches that of commercially available $5 \mathrm{~kW}$ thrusters. Thrust, specific impulse, efficiency, and plasma parameter profiles in the plume are presented in a previous work. ${ }^{16}$ The thruster was operated at $300 \mathrm{~V}$ and either 5.4 or $10 \mathrm{~A}$.

\section{Results}

The initial phase of this experiment was the characterization of the HARP system's performance. Figure 10 shows a representative plot of position, velocity, and acceleration 


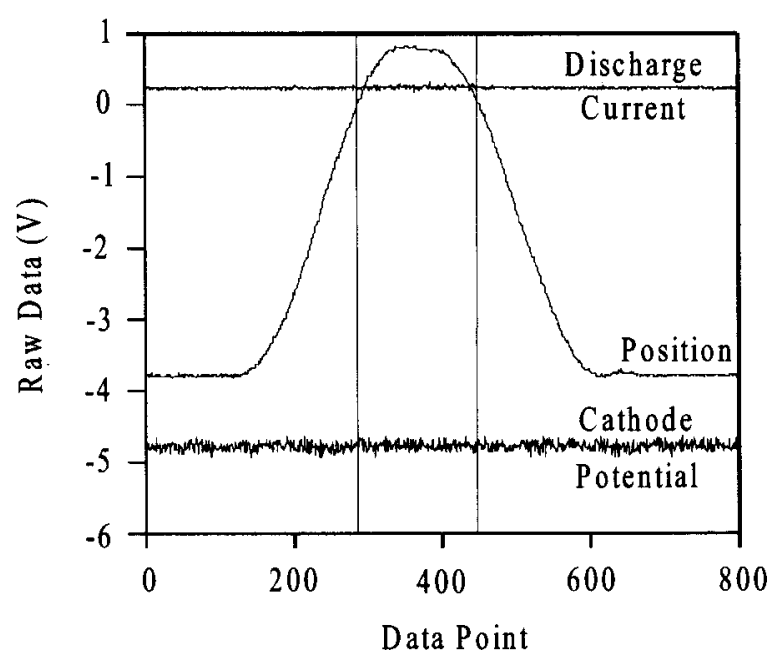

FIG. 11. Discharge current and cathode potential profiles during probe sweep. The vertical lines indicate the exit plane location.

versus time. From the plot, it can be determined that the total residence time inside the chamber is approximately $80 \mathrm{~ms}$. This time varied slightly from stroke to stroke but always remained under $100 \mathrm{~ms}$, well below the predicted $150 \mathrm{~ms}$ ablation time. The plot also shows the maximum velocity 3 $\mathrm{m} / \mathrm{s}$ and acceleration $6 \mathrm{~g}$ that the system achieved.

Once the performance of the HARP system was confirmed, it was installed in the LVTF and measurements were attempted with the triple probe described above. Subsequent analysis showed the data from the triple probe to be suspect

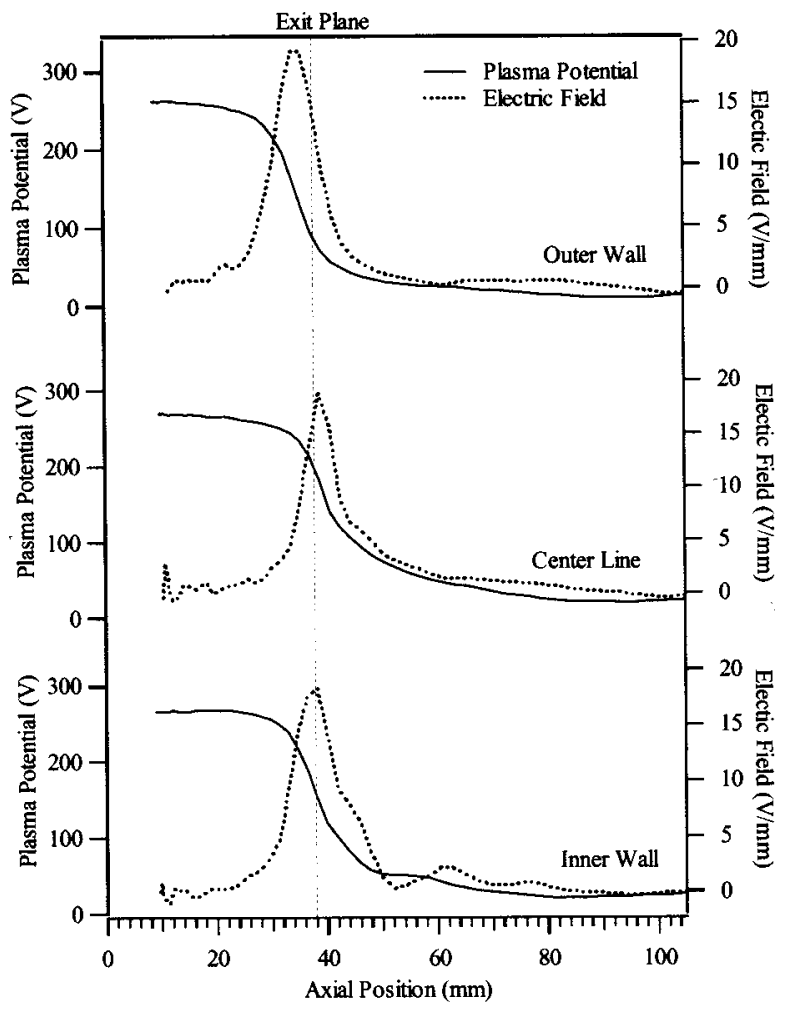

FIG. 12. Plasma potential and axial electric field profiles at three representative radial locations for the $300 \mathrm{~V}, 10 \mathrm{~A}$ case. The anode face corresponds to a position of $0 \mathrm{~mm}$ and the exit plane, as marked, is at a position of 38 $\mathrm{mm}$.

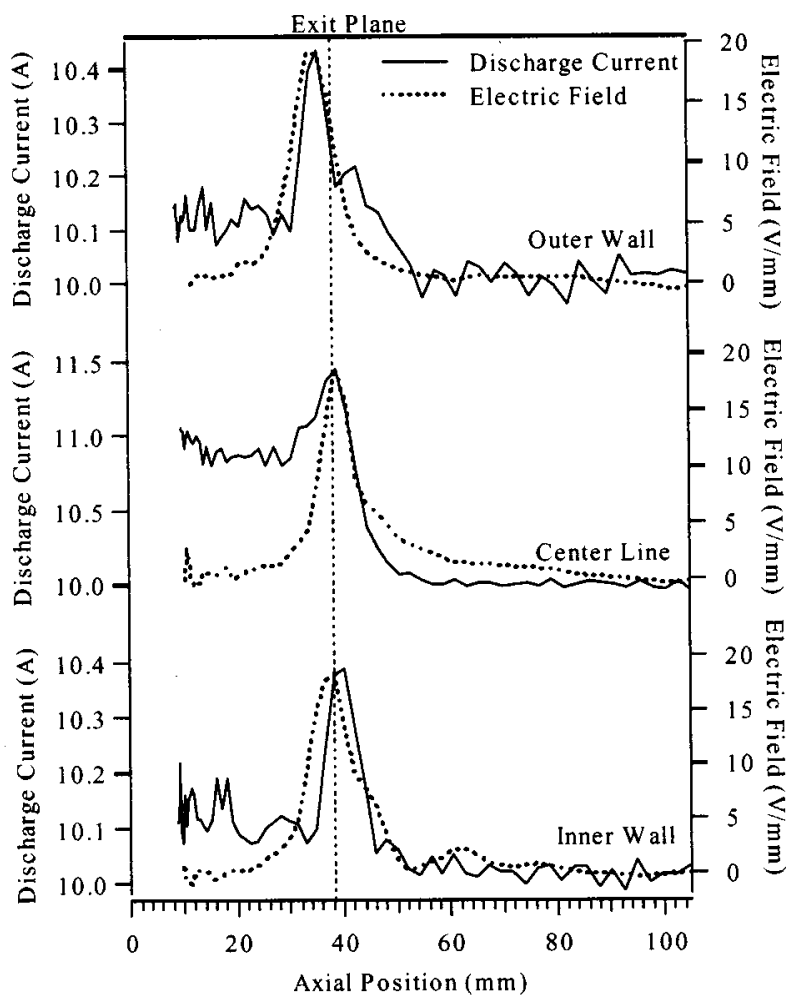

FIG. 13. Perturbations in the discharge current as a function of probe position are compared to the axial electric field profiles.

due to sheath interaction between the individual electrodes. However, discharge current, cathode potential, and probe position data were successfully collected and are presented in Fig. 11. These data clearly show the ability of the HARP system to insert and remove electrostatic probes while significantly reducing, if not eliminating, perturbation to the thruster. Note that the configuration of the triple probe approximated the "dummy" probe used in the AFRL perturbation test.

The results of the triple probe were very encouraging but it remained to be shown that the HARP system could return accurate measurements while not perturbing the thruster. The triple probe was removed and the floating emissive probe was attached to the high-speed actuator. Because of the results of the previous experiment, the thruster parameters were not monitored during the test at the same thruster condition of $300 \mathrm{~V}$ and $5.4 \mathrm{~A}$. However, discharge current data were collected with plasma potential at a second condition of 10 A. These data are presented in Fig. 12 and 13. For this case, the cathode potential with respect to ground was $-23 \mathrm{~V}$, therefore the anode was at a potential of $277 \mathrm{~V}$ with respect to ground. The plasma potential measurements of Fig. 12 show that the potential remains nearly constant over the first $75 \%$ of the channel. As expected, a sharp drop occurs between 30 and $40 \mathrm{~mm}$ indicating the location of the main acceleration region. The axial electric field profiles show clearly the location of maximum acceleration at 35.5 $\mathrm{mm}, 2.5 \mathrm{~mm}$ upstream of the exit plane. These data indicate that the acceleration region extends $2-3 \mathrm{~cm}$ downstream of the exit plane, which agrees quite well with independent laser-induced fluorescence $(\mathrm{LIF})^{17}$ data taken on the same 
thruster at the same conditions. The data also agree with potential measurements from other Hall thrusters. ${ }^{13}$ Note that the profile remains uniform across the width of the discharge chamber, which is also consistent with LIF data. ${ }^{17}$

From Fig. 13, it can be seen that the thruster was perturbed, however, worse case variations in the discharge current did not exceed approximately $15 \%$. For most cases, the discharge current was perturbed by less than $10 \%$. This is well below what was observed in previous experiments (Fig. 2) when the probe was obviously ablating. Moreover, it is also much smaller than perturbations observed by other researchers conducting similar experiments. ${ }^{13,15}$ For example, Hargus $^{13}$ observed discharge current perturbations of $150 \%$ and completely destroyed his probe. Upon examination of the probe following this test, there was no noticeable material ablation such as that illustrated in Fig. 3 or as seen by Hargus.

The results of the emissive probe experiment yielded some rather unexpected insights. Referring back to Fig. 13, perturbations to the discharge current are seen to correspond very well to the spatial location of the peak axial electric field. Recall that power deposition to the probe is proportional to the third power of the drift velocity, while the $\mathbf{E} \times \mathbf{B}$ drift velocity is proportional to the magnitude of the electric field. Therefore, it is expected that the probe would experience the greatest heat load where the electric field has its largest magnitude. It follows immediately that this would be the point of greatest probe material ablation and hence discharge current perturbation. However, if this were the only mechanism disturbing thruster operation, once the probe was inserted into the discharge chamber, a portion of the probe would always receive this heat load, and probe material would continue to ablate. In this case, the discharge current would remain artificially high while the probe was present inside the discharge chamber. This would also be the case if the probe were simply acting as a physical barrier, impeding the motion of the electrons as they drifted azimuthally inside the thruster. The results of the emissive probe imply that the presence of the conductor, spatially coincident with the peak electric field, causes the greatest perturbation to the thruster. It should be noted that this is also the location of maximum radial magnetic field strength and hence lowest electron axial mobility. The data suggest that the conductor is acting as a low-impedance path between magnetic field lines, causing an increase in electron diffusion to the anode. This would appear as an artificial increase in the thruster discharge current. Referring back to the triple probe results, in this case the flush-mounted conductor had effectively no length in the axial direction and therefore would not have provided the same path between magnetic field lines. This would explain why there was no apparent change in the discharge current while the triple probe was in the thruster channel.

The above results have some potentially serious implications for future Hall thruster experiments. If it is verified that the conductor is enhancing electron diffusion across the magnetic field lines to the anode, it may not be possible to probe the discharge chamber using any type of finite length electrostatic probe. Planar probes might still be a viable solution, however, very little planar probe theory exists for the conditions encountered in the Hall thruster.

\section{ACKNOWLEDGMENTS}

The research contained herein was sponsored by the Air Force Office of Scientific Research; this support is gratefully acknowledged. In addition the authors would also like to thank their colleagues at the Air Force Research Laboratory and PEPL for their useful insights and suggestions and the Moscow Aviation Institute (MAI) for providing the hollow cathode. J. H. is supported under the Air Force Palace Knight program.

${ }^{1}$ H. M. Mott-Smith and I. Langmuir, Phys. Rev. 28, 727 (1926).

${ }^{2}$ T. L. Rhodes, Ch. P. Ritz, R. D. Bengtson, and K. R. Carter, Rev. Sci. Instrum. 61, 3001 (1990).

${ }^{3}$ W. Seifert, D. Johanning, H. R. Lehmann, and N. Bankov, Contrib. Plasma Phys. 26, 237 (1986).

${ }^{4}$ C. M. Marrese, J. M. Haas, M. T. Domonkos, A. D. Gallimore, S. Tverdoklebov, and C. Garner, AIAA Pap. 96, 2969 (1996).

${ }^{5}$ A. D. Gallimore, M. L. Reichenbacher, C. M. Marrese, S.-W. Kim, and J. E. Foster, AIAA Paper 94, 3137 (1994).

${ }^{6}$ S-W. Kim, J. E. Foster, and A. D. Gallimore, AIAA Pap. 96, 2972 (1996).

${ }^{7}$ P. T. C. De Boer, J. Propul. Power 12, 783 (1996).

${ }^{8}$ R. L. Burton, S. G. DelMedico, and J. C. Andrews, J. Propul. Power 9, 771 (1993).

${ }^{9}$ G. Guerrini, C. Michaut, M. Dudeck, A. N. Vesselovzorov, and M. Becal, IEPC Paper 97-053, Sept. 1997.

${ }^{10}$ D. L. Tilley, A. J. Kelly, and R. G. Jahn, AIAA Pap. 90, 2667 (1990).

${ }^{11}$ R. L. Burton, J. Propul. Power 12, 1099 (1996).

${ }^{12}$ J. M. Haas, G. Spanjers, K. McFall, and R. Spores, AIAA Pap. 98, 3656 (1998)

${ }^{13}$ W. A. Hargus and M. A. Cappelli, AIAA Pap. 99, 2721 (1999).

${ }^{14}$ J. Boedo, D. Gray, L. Chousal, and R. Conn, Rev. Sci. Instrum. 69, 2663 (1998).

${ }^{15}$ M. T. Domonkos, C. M. Marrese, J. M. Haas, and A. D. Gallimore, AIAA Pap. 97, 3062 (1997).

${ }^{16}$ J. M. Haas, F. S. Gulczinski, A. D. Gallimore, G. Spanjers, and R. Spores, AIAA Pap. 98, 3503 (1998).

${ }^{17}$ G. J. Williams, T. B. Smith, F. S. Gulczinski, B. E. Beal, A. D. Gallimore, and R. P. Drake, AIAA Pap. 99, 2424 (1999). 